UDC: $519.6: 519.147$

\title{
Linear and nonlinear optimization models of multiple covering of a bounded plane domain with circles
}

\author{
Sh. I. Galieva ${ }^{\text {a }}$ A. V. Khorkov ${ }^{b}$ \\ Tupolev Kazan National Research Technical University-KAI, \\ 10 ul. Karla Marksa, Kazan, 420111, Russia \\ E-mail: ${ }^{a}$ sh.galiev@mail.ru, ${ }^{b}$ aLex22fcrk@yandex.ru
}

Received 30.05.2019.

Accepted for publication 14.11.2019.

\begin{abstract}
Problems of multiple covering ( $k$-covering) of a bounded set $G$ with equal circles of a given radius are well known. They are thoroughly studied under the assumption that $G$ is a finite set. There are several papers concerned with studying this problem in the case where $G$ is a connected set. In this paper, we study the problem of minimizing the number of circles that form a $k$-covering, $k \geq 1$, provided that $G$ is a bounded convex plane domain.

For the above-mentioned problem, we state a 0-1 linear model, a general integer linear model, and a nonlinear model, imposing a constraint on the minimum distance between the centers of covering circles. The latter constraint is due to the fact that in practice one can place at most one device at each point. We establish necessary and sufficient solvability conditions for the linear models and describe one (easily realizable) variant of these conditions in the case where the covered set $G$ is a rectangle.

We propose some methods for finding an approximate number of circles of a given radius that provide the desired $k$-covering of the set $G$, both with and without constraints on distances between the circles' centers. We treat the calculated values as approximate upper bounds for the number of circles. We also propose a technique that allows one to get approximate lower bounds for the number of circles that is necessary for providing a $k$-covering of the set $G$. In the general linear model, as distinct from the $0-1$ linear model, we require no additional constraint. The difference between the upper and lower bounds for the number of circles characterizes the quality (acceptability) of the constructed $k$-covering.

We state a nonlinear mathematical model for the $k$-covering problem with the above-mentioned constraints imposed on distances between the centers of covering circles. For this model, we propose an algorithm which (in certain cases) allows one to find more exact solutions to covering problems than those calculated from linear models.

For implementing the proposed approach, we have developed computer programs and performed numerical experiments. Results of numerical experiments demonstrate the effectiveness of the method.

Keywords: linear models of the multiple covering problem, $k$-covering of a bounded set, nonlinear models of the $k$-covering problem with circles of a given radius, solvability conditions for linear models of the $k$-covering problem
\end{abstract}

Citation: Computer Research and Modeling, 2019, vol. 11, no. 6, pp. 1101-1110.

(C) 2019 Shamil I. Galiev, Alexander V. Khorkov 


\section{Introduction}

Let $G$ be a bounded convex closed set with nonempty interior in a plane $P$ and let $S=$ $=\left\{K_{1}, K_{2}, \ldots, K_{m}\right\}$ be a set of closed circles of a given radius $r$. We say that circles of this set form a $k$-fold covering (a $k$-covering), $k \geq 1$, of the set $G$ if each point $s$ in $G$ belongs to at least $k$ circles in $S$.

The $k$-covering problems for plane domains have many applications, in particular, in finding locations of mobile, navigation, and ambulance stations. One also encounters covering problems when projecting sensor systems for monitoring the infrastructure of telecommunication networks, environmental control systems, industrial equipment monitoring systems, smart city systems and so on (see [Ammari, 2009; Erzin, Astrakov, 2011; Huang, Tseng, 2005; Farahani et al., 2012; Peixoto, Costa, 2017; Wang, 2011; Yeasmin, 2014]). In [Zhang et al., 2009] it was noted that "Wireless sensor networks (WSNs) are ideal candidates for monitoring the physical space and enabling a variety of applications...". In sensor networks, to compensate for the failure of some sensors, one provides a $k$-covering of a given area with $k \geq 2$.

There are many papers devoted to covering problems for certain bounded plane domains such as a square, a rectangle etc. (see [Nurmella, 2000; Suzuki, Drezner, 2009] and references therein). Multiple coverings of bounded domains are studied, for example, in [Galiev, Karpova, 2010; Tabirca et al., 2013] and in papers referred to therein. In most works on covering problems one minimizes, as a rule, the radii of covering circles. In practice, the radii of covering circles are given and it is necessary to find their minimum number. Based on practical problems, we assume that the radii of covering circles are given.

Note that the covering problem considered is NP-hard (see, for example, [Culberson, Reckhow, 1994; Garey, Jonson, 1979; Megiddo, 1984; Khachai, Poberiy, 2013]).

In this paper, to solve the $k$-covering problem for a set $G$, we construct a rectangular grid with the step $\Delta$, whose nodes form a finite set $T_{\Delta}$. This allows us to reduce the $k$-covering problem $(k \geq 1)$ for the set $G$ with circles of given radii either to a 0-1 linear programming (LP) problem or to a general integer LP problem.

We establish necessary and sufficient solvability conditions for linear models. In the case where the covered set $G$ is a rectangle, we propose one (easily realizable) variant of these conditions. We also propose techniques for finding an approximate number of circles of radius $r$ that provide a $k$-covering of the set $G$ and determine the locations of their centers, both with and without constraints imposed on distances between the circles' centers. We treat the calculated values as approximate upper bounds for the number of circles.

We propose a method which allows one to find approximate lower bounds for the number of circles that is necessary for providing a $k$-covering of a (nearly arbitrary) domain $G$. The difference between the upper and lower bounds for the number of circles characterizes the quality (acceptability) of the covering.

We state a nonlinear mathematical model of the $k$-covering problem with the above-mentioned constraint imposed on the minimum distance between the centers of covering circles. We propose an algorithm that (in certain cases) allows one to find solutions to the covering problems that are more precise than those calculated from the linear models.

To implement the proposed approach, we have developed computer programs and performed numerical experiments. Results of numerical experiments demonstrate the effectiveness of the method. 


\section{Linear mathematical models of the problem}

Choose the step size $\Delta$ and construct a rectangular grid on the set $G$ with the step $\Delta x=\Delta y=\Delta$. Denote the collection of grid nodes that belong to $G$ as $T_{\Delta}=\left\{t_{1}, \ldots, t_{n}\right\}, t_{i} \in G$. Evidently, the $T_{\Delta}$ is finite. Let us add some points on the domain boundary to the set $T_{\Delta}$ [Galiev, Khorkov, 2019]. Assume that $G, k(k \geq 1), \Delta$ and $r$ are given, and the finite set $T_{\Delta}$ is constructed. Consider the following problems.

Problem P1. Find a $k$-covering $(k \geq 1)$ of the set $G$ with circles of a given radius $r$ so as to minimize the number of covering circles and determine the locations of their centers in $G$.

Problem P2. Find a $k$-covering of the set $G$ with circles of radius $r$ so as to minimize the number of covering circles and determine the locations of their centers under the condition that the center of each covering circle should coincide with some point in the set $T_{\Delta}$, while each point in $T_{\Delta}$ should coincide with at most one of the circles' centers.

Problem P3. Find a $k$-covering of the set $T_{\Delta}$ with circles of radius $r$ so as to minimize the number of covering circles and determine the locations of their centers and to locate the center of each covering circle in $T_{\Delta}$ in such a way that each point in $T_{\Delta}$ coincides with at most one of the circles' centers.

To establish solvability conditions for linear models of $k$-covering problems, we mention some results obtained in [Galiev, Khorkov, 2019]. Let the symbol $d(s, t)$ stand for the Euclidean distance between points $s$ and $t$ in the plane $P$. Choose $\Delta$ and construct the set $T_{\Delta}$ on $G$. Introduce the parameter $\alpha, 0<\alpha<r$, and the matrix $A=\left(a_{i j}\right)$, where

$$
a_{i j}= \begin{cases}1, & \text { if } \quad d\left(t_{i}, t_{j}\right) \leq r-\alpha, \\ 0, & \text { if } \quad d\left(t_{i}, t_{j}\right)>r-\alpha .\end{cases}
$$

Let us define the vector $Z$ with coordinates $z_{1}, \ldots, z_{n}$, where $z_{i}$ is the number of circles of radius $r-\alpha$ whose centers coincide with the point $t_{i}, 1 \leq i \leq n$. Let the symbol $K$ stand for the $n$-dimensional vector each component of which equals $k$. Consider the system

$$
z_{1}+z_{2}+\cdots+z_{n} \rightarrow \min
$$

subject to

$$
\begin{gathered}
A Z \geq K, \\
z_{i} \in\{0,1\}, \quad 1 \leq i \leq n .
\end{gathered}
$$

The constructed problem (1)-(3) with $\alpha=0$ is the problem of $k$-covering of $T_{\Delta}$ with the least possible number of circles of radius $r$ such that the circles' centers coincide with some points in $T_{\Delta}$, while each point in $T_{\Delta}$ coincides with at most one of the circles' centers. Therefore, system (1)-(3) solves Problem P3.

It is clear that the model considered allows one to locate sensors at various points of the set $T_{\Delta}$. At the same time, the above-mentioned system is studied insufficiently, in particular, it is necessary to find out when it is solvable and how the values $k, r$, and $\Delta$ correlate.

In [Galiev, Khorkov, 2019], we prove that one can find an approximate solution to Problem P2 by reducing the radii of covering circles by the value $\alpha_{0}=\Delta \sqrt{2} / 2$ and solving Problem P3 for circles of radius $r-\alpha_{0}$. Then we obtain a $k$-covering of the set $G$ with just obtained locations of the circles' centers and the initial radius $r$. We can replace $\alpha_{0}$ with arbitrary $\alpha, \alpha_{0} \leq \alpha \leq 2 \alpha_{0}$.

We treat an approximate solution of Problem P2 as an approximate solution of Problem P1.

If we allow the centers of some circles to coincide, then we should replace (3) with the following new constraint:

$$
z_{i} \in Z^{+}, \quad 1 \leq i \leq n, \quad Z^{+}=\{0,1,2, \ldots\} .
$$


In this case, system (1),(2) and (4) solves the problem which differs from Problem P3 in that the condition "each point in $T_{\Delta}$ coincides with at most one of the circles' centers" is not stated. We refer to problems $\mathrm{P} 2$ and $\mathrm{P} 3$ without the above-mentioned condition as problems $\mathrm{P} 2 *$ and $\mathrm{P} 3 *$, respectively.

Let us impose constraints on the distances between the circles' centers. At present, apart from our paper mentioned above, there are very few studies where one imposes constraints on the distances between the centers of covering circles (see [Astrakov, 2017; Kim et al., 2009]); unfortunately, no numerical results (suitable for comparison) are described there. Let us require that the minimum distance between the circles' centers be not less than some given value $\lambda$. Using results obtained in [Galiev, Lisafina, 2013], we impose the following constraints (see [Galiev, Khorkov, 2019] for details):

$$
B Z \leq P^{*}
$$

Constraints (5) guarantee that the distances between the circles' centers are not less than $\lambda$. Consequently, by solving problem (1)-(3), (5) or problem (1), (2), (4), and (5), we determine the locations of the circles' centers, taking into account the constraints imposed on the distances between their centers.

The stated problems are integer LP problems. If they are solvable, then one can solve them by any integer LP method (in this paper, we describe the results obtained with the help of the CPLEX12.6.3 library). The decrease in the grid step $\Delta$ leads to an increase in the dimension $n$ of the problems considered and makes their solution more difficult. If a large-dimension problem does not include constraint (5) (but only (3)), then one can solve this problem using the heuristic algorithm proposed in [Galiev, Khorkov, 2019].

\section{Solvability conditions for stated LP problems}

Let the circle radius and the covering multiplicity in problems P2 and P3 be given. We have to propose a way to choose the value of the parameter $\Delta$ so as to make system (1)-(3) solvable with given $G, k$, and $r$. Note that a grid on $G$ can be rectangular, oblique, or have some other shape. Regardless of the way used for the grid construction, we obtain a finite set $T_{\Delta}$, which is important for stating system (1)-(3) for problems P2 and P3. One can easily see that the following condition is necessary and sufficient for the solvability of problem (1)-(3).

Condition U1: System (1)-(3) is solvable if and only if for any point $t_{j}$ in $T_{\Delta}$ there exist at least $k$ various points $t_{i}$ in $T_{\Delta}$ whose distance from $t_{j}$ is at most equal to the radius of the circles that cover $T_{\Delta}$.

The verification of Condition U1 is a nontrivial task, therefore we propose an easily verifiable variant of these conditions for the case where the covered set $G$ is a rectangle. Let $G$ be a rectangle, denote it by $G_{t}$. Assume that the lengths $a$ and $b$ of its sides are multiples of the value $\Delta$. For an arbitrary point $s, s \in G_{t}$, we let $R(s)$ denote the closed circle of radius $r$ centered at the point $s$. Assume that $s$ is a vertex of the rectangle $G_{t}$, consequently, $s$ coincides with some point in $T_{\Delta}$. Then with $r=\Delta$ the set $G_{t} \cap R(s)$ contains 3 distinct points that belong to $T_{\Delta}$, and with the radius $r=\Delta \sqrt{2}$ the set $G_{t} \cap R(s)$ contains 4 distinct points that belong to $T_{\Delta}$ and so on. For any other point $s$ in $T_{\Delta}$ the number of various points in $T_{\Delta}$ that belong to $G_{t} \cap R(s)$ is not less than that in the case where $s$ is a vertex of the rectangle. Therefore, we conclude that Condition U1 is fulfilled if $r$ and $\Delta$ satisfy the following relations: if $\Delta=r$, then $1 \leq k \leq 3$; if $\Delta=r / \sqrt{2}$, then $1 \leq k \leq 4$; if $\Delta=r / 2$, then $1 \leq k \leq 6$; if $\Delta=r / 2 \sqrt{2}$, then $1 \leq k \leq 9$, and in what follows the covering multiplicity $k$ and the value $\Delta$ are related by $R_{\Delta}=r$, where $R_{\Delta}$ is the radius of the circle centered at the vertex of $G_{t}$, and this circle contains $k$ points that belong to $T_{\Delta}$. 
Choosing various values of $\Delta$ and $r$, we obtain various numbers of points in the set $G_{t} \cap R(s)$. Consequently, we conclude that the number of these points in $G_{t} \cap R(s)$ determines the maximum possible value of the multiplicity of the covering of the whole rectangle $G_{t}$. The number of points in $T_{\Delta}$ depends on dimensions of the rectangle and can be very large; however, the mathematical model (1)-(3) is solvable only when the covering multiplicity depends on the number of points in $G_{t} \cap R(s)$. Therefore, we conclude that the value of the multiplicity in system (1)-(3) cannot be arbitrary.

The constraint imposed on the minimum distance between the circles' centers also affects the solvability of the stated LP problem. Evidently, for a 1-fold covering, the minimum distance between the circles' centers $(\lambda)$ cannot exceed the diameter of covering circles, that is, $\lambda \leq 2 r$; if the covering multiplicity is greater than 1 , then $\lambda \leq r$. Denote the set of points in $T_{\Delta}$ that belong to $G_{t} \cap R(s)$ by $T_{\Delta}^{t}$. The number of these points defines the maximum multiplicity of the covering of the rectangle $G_{t}$. Since the minimum distance between points cannot be less than the value $\lambda$, we solve the problem of packing the largest number of circles of radius $\lambda / 2$ whose centers belong to $T_{\Delta}^{t}$. As a result, we calculate the number $k^{*}$ of circles whose centers belong to $T_{\Delta}^{t}$. In particular, if $\lambda=\Delta$, then the centers of packed circles belong to $T_{\Delta}^{t}$, consequently, $k^{*}=k$; if $\lambda=r / 2$, then the number of packed circles of radius $\lambda / 2$ whose centers belong to $T_{\Delta}^{t}$ equals 6 or 7 depending on the value $\Delta$. Therefore, constraints imposed on the distances between the circles' centers affect the maximum possible value of the covering multiplicity. Consequently, if we add constraint (5) to system (1)-(3), then the resulting system is solvable, provided that the covering multiplicity does not exceed the maximum number of (packed in $G_{t}$ ) circles of radius $\lambda / 2$ whose centers belong to $T_{\Delta}^{t}$. One can state the above-mentioned packing problem as an LP problem (see [Galiev, Lisafina, 2013]); moreover, the resulting LP problem is easily solvable, because the number of points in the set $T_{\Delta}^{t}$ is less than that in the set $T_{\Delta}$.

\section{Approximate lower bounds for the number of circles in a $k$-covering}

Note that covering and $k$-covering problems are being widely studied in the literature and establishing bounds for the objective function is a quite nontrivial task.

Choose $r, \Delta, k(1 \leq k \leq 4), \lambda \geq 3 \Delta$ and put $\beta=\Delta \sqrt{2}$. Introduce the following notation:

- let the symbol $n_{\text {opt }}$ stand for the minimum number of circles of radius $r$ that form a $k$-covering of the set $G$, provided that the minimum distance between their centers is not less than $\lambda$;

- let the symbol $n_{1}$ stand for the minimum number of circles of radius $r+\beta$ obtained as a solution to problem (1)-(3) (which forms a $k$-covering of $T_{\Delta}$ ).

In [Galiev, Khorkov, 2019] we prove that $n_{1} \leq n_{\text {opt }}$, consequently, one can find a lower bound for the number of circles obtained as a solution to Problem P1 by solving Problem P3. Solving Problem P3, we get an upper bound for the number $n_{\text {opt }}$; denote it by $n_{2}$. As a result, we get the relation $n_{1} \leq n_{\text {opt }} \leq n_{2}$. The difference $n_{2}-n_{1}$ defines the segment that contains the estimated value $n_{\text {opt }}$.

Let us now consider problems $\mathrm{P} 2 *$ and $\mathrm{P} 3 *$ whose solution does not require that each point in $T_{\Delta}$ coincide with at most one of the admissible centers of the circles. In this case, the mathematical model of Problem P3* takes the form (1), (2), and (4). For arbitrary $\Delta, \Delta>0$, Problem P3* is solvable with any values of the covering multiplicity, because one of the admissible solutions to this problem consists in placing $k$ centers of covering circles at each point of the set $T_{\Delta}$. To solve Problem P2*, we reduce the radius of covering circles by $\alpha_{0} \geq \Delta \sqrt{2} / 2$ and seek for a covering with circles of radius $r-\alpha_{0}$. One can easily see that this problem is solvable for any values of the covering multiplicity $(k)$, provided that $\alpha \geq \Delta \sqrt{2} / 2$ (for a rectangular grid). Therefore, condition U1 becomes extra here. Having solved 
the stated Problem P2*, in other words, having determined the number of circles and locations of their centers, we assume that the circles have the initial radius $r$. The obtained locations of the centers of circles of radius $r$ form the desired $k$-covering of the set $G$.

In [Galiev, Khorkov, 2019], we obtain approximate lower bounds for the number of circles in a $k$-covering of $G$ under one additional assumption. Namely, we require that the minimum distance between the circles' centers be not less than $3 \Delta$. For problems P2* and P3* such assumptions are not necessary, because any point of the set $T_{\Delta}$ can coincide with more than one circle's center. We obtain approximate lower bounds for the number of circles in a $k$-covering of $G$ in the following way. Choose $\Delta, \Delta>0$, construct $T_{\Delta}$, and solve system (1), (2) and (4) (Problem P3*), when $\alpha=0$, while the circle radius equals $r+\Delta \sqrt{2}$. As a result, we get the minimum number $\left(n_{1}^{*}\right)$ of circles of radius $r+\Delta \sqrt{2}$ that form a $k$-covering of $T_{\Delta}$. Let $n_{\text {opt }}^{*}$ be the minimum number of circles of radius $r$ that form a $k$-covering of the set $G$. Similarly to Theorem 2 in [Galiev, Khorkov, 2019], one can prove that $n_{1}^{*} \leq n_{\text {opt }}^{*}$.

To obtain an upper bound for $n_{\text {opt }}^{*}$, we solve Problem P3* for circles' radii smaller than $r$. To this end, we consecutively put $\Delta:=\Delta / 2^{m}\left(\alpha:=\alpha / 2^{m}\right)$, construct $T_{\Delta / 2^{m}}, m=0,1,2,3, \ldots$, and then solve Problem (1), (2), and (4) with $\alpha / 2^{m}$ in place of $\alpha$. As a result, we consecutively calculate the number $n_{\alpha / 2^{m}}^{*}$ of circles of radius $r-\alpha / 2^{m}$ that form a $k$-covering of the set $T_{\Delta / 2^{m}}$, while

$$
n_{\alpha}^{*} \geq n_{\alpha / 2}^{*} \geq n_{\alpha / 2^{2}}^{*} \geq \ldots \geq n_{\alpha / 2^{m}}^{*} \geq \ldots
$$

In this sequence, starting with some $m$, all numbers $n_{\alpha / 2^{m+j}}$ coincide for any $j \geq 1$; denote $n_{\alpha / 2^{m+j}}=M$. If the radii of these circles are equal to $r$, then these circles form a $k$-covering of the set $G$. Evidently, $n_{\alpha / 2^{m}} \geq n_{o p t}^{*}$. Therefore, for values $n_{\alpha / 2^{j}}^{*}$ that are less than $n_{\alpha / 2^{m}}^{*}$ we obtain the following upper bound for $n_{o p t}^{*}: n_{1}^{*} \leq n_{\text {opt }}^{*} \leq n_{\alpha / 2}^{*}$. The difference of values $n_{1}^{*}$ and $n_{\alpha / 2}^{*}$ (that can be calculated) characterizes the acceptability of the bound for $n_{o p t}^{*}$.

\section{The nonlinear model of the covering problem}

Consider the problem of the $k$-covering of the set $G$ with a given number $(N)$ of equal circles with the minimum possible radius $r_{\text {min }}$. Denote the center of the circle $K_{j}$ by $c_{j}$ and the coordinates of the center $c_{j}$ by $x_{j}, y_{j}, 1 \leq j \leq N$. Put $u=\left(x_{1}, y_{1}, \ldots, x_{N}, y_{N}\right)$. The calculation of $r_{\min }$ is reduced to the evaluation of the following expression (see [Galiev, 1994]):

$$
r_{\min }=\min _{u} \max _{s \in G} \min _{j \in J_{K}} d\left(s, c_{j}\right),
$$

where $J_{k}$ is the set of subscripts of the centers $c_{j}$ of $N-k+1$ circles, whose distances from $s$ are not less than that of the remaining $k-1$ centers among $c_{j}, 1 \leq j \leq N$. The calculation of $r_{\min }$ is a nonsmooth optimization problem, one can solve it by known nondifferentiable optimization methods. Making use of the properties of these problems, in [Galiev, 1994] we propose algorithms that allow one (at least) not to increase the radius on each step.

For chosen $N$ in the segment $\left[n_{1}, n_{2}\right]$, we assume that an approximate value $\widetilde{r}$ of radius $r_{\text {min }}$ is already calculated. We compare it with the circle radius $r$ in Problem P1. If it appears that $\widetilde{r} \leq r$, then $N$ circles of radius $\widetilde{r}$ form a $k$-covering of the set $G$. Then, evidently, $N$ circles of radius $r$ also form a $k$-covering of the set $G$. Therefore, the minimum number of circles that form a $k$-covering of the set $G$ belongs to the segment $\left[n_{1}, N\right]$.

One can calculate the value $r_{\text {min }}$ by formula (6), using any algorithm proposed in [Galiev, 1994]. Imposing no constraint on the distances between the circles' centers, in this paper we use Algorithm IV proposed in the work mentioned above. In the case where the problem statement includes the indicated 
constraints, we use the modification of Algorithm V described in the above-mentioned paper. First of all, we introduce the following definition: follows:

A $k$-multiple Voronoi-Dirichlet domain $D_{j}^{k}$ for a set of points $\left\{c_{j}, 1 \leq j \leq N\right\}$ is defined as

$$
D_{j}^{k}=D_{j}^{k}(u)=\left\{s \in G: d\left(s, c_{j}\right) \leq \min _{i \in J_{k}} d\left(s, c_{j}\right)\right\} .
$$

In the case of a constraint imposed on the minimum distance between the circles' centers, it is necessary to introduce an additional condition (along with condition (6)), namely,

$$
d\left(c_{i}, c_{j}\right) \geq \lambda, \quad 1 \leq i, \quad j \leq N, \quad i \neq j .
$$

To calculate (with fixed $N$ ) the value $\widetilde{r}$ in accordance with (6) and (7), we use the following modification of Algorithm V.

Algorithm $V^{*}$ :

1. Choose initial locations of the centers of $N$ circles $K_{j}, 1 \leq j \leq N$. The coordinates of the centers of these circles form the vector $u=\left(x_{1}, y_{1}, \ldots, x_{N}, y_{N}\right)$. One can obtain this initial location of the circles' centers satisfying conditions (7) from the solution to the LP problem (1), (2), (4), when the number of circles exceeds $N$, arbitrarily dropping extra points.

2. On the set $G$, construct $k$-multiple Voronoi-Dirichlet domains $D_{j}^{k}$ for the set of points $\left\{c_{j}, 1 \leq j \leq N\right\}$.

3. Among elements $c_{j}, 1 \leq j \leq N$, choose $c_{i}$ which is at the maximal distance from the farthest vertex $v$ of the set $D_{i}^{k}$. Let $c_{i}^{*}$ be the center of the minimum circle that contains $D_{i}^{k}$. In the segment $\left[c_{i}, c_{i}^{*}\right]$ choose a point $b_{i}$ such that it is at the maximal distance from $c_{i}$ and all points of the segment $\left[c_{i}, c_{i}^{*}\right]$ satisfy inequalities (7). If, in addition, this point $c_{i}^{*}$ coincides with $c_{i}$, then choose the next point $c_{l}$ in $\left\{c_{1}, \ldots, c_{N}\right\} \backslash\left\{c_{i}\right\}$ and repeat the search for an analogous point $b_{l}$. The point $b_{i}$ obtained (different from $c_{i}$ ) together with the set $\left\{c_{1}, \ldots, c_{N}\right\} \backslash\left\{c_{i}\right\}$ generate a new vector $u^{*}$.

4. If the Euclidean distance between $u$ and $u^{*}$ does not exceed given $\epsilon>0$, then stop, otherwise put $u=u^{*}$ and go to step 2 .

Note that the radius of the circles that form a $k$-covering of $G$, at least, does not increase on each step of this algorithm.

As a drawback of the described algorithm, we mention the fact that it does not guarantee that the solution obtained provides the global minimum value of the circle radius $r_{\min }$. For finding the global minimum, one can make use of the Lipschitz property of the objective function (one can prove that the function in (1) is Lipschitz for the above-mentioned $J_{k}$ ), but we do not do it in this paper.

\section{Numerical results}

Solvability conditions for the stated linear models of covering problems depend on the set $G$, the varying parameters of mathematical models, and the grid step. Constructing the grid in various ways (as a rectangular or oblique one), varying the grid step $\Delta$ and the radius $r$ of covering circles, we obtain various numbers of points in $T_{\Delta}$ that belong to the set $G \cap R(s)$, and the number of these points in $G \cap R(s)$ defines the maximum possible value of the covering multiplicity for the whole given set $G$ in problems $\mathrm{P} 2$ and $\mathrm{P} 3$.

In Table 1, we give maximum values of the covering multiplicity $(k)$ with which the system (1)-(3) is solvable depending on the chosen domain (a rectangle $G_{t}$ or an equilateral triangle $\mathrm{Tr}$ ) 
Table 1. Maximum values of the covering multiplicity, with which the system (1)-(3) is solvable

\begin{tabular}{|c|l|l|l|l|l|l|l|l|l|}
\hline The covered set & \multicolumn{7}{|c|}{ The radius of covering circles $r=m \times \Delta$} \\
\cline { 2 - 11 } & $\Delta$ & $2 \Delta$ & $3 \Delta$ & $10 \Delta$ & $20 \Delta$ & $40 \Delta$ & $60 \Delta$ & $80 \Delta$ & $100 \Delta$ \\
\hline$G_{t}$ & 3 & 6 & 11 & 90 & 335 & 1297 & 5101 & 6452 & 7995 \\
\hline$T r$ & 3 & 6 & 10 & 72 & 264 & 1010 & 2237 & 3953 & 5300 \\
\hline
\end{tabular}

and radius $r$. In this table, the value $\Delta$ equals 0.01 , while the radius $r$ of covering circles takes the form $r=m \times \Delta, 1 \leq m \leq 100$. For the rectangle, we construct a rectangular grid, while in the case of an equilateral triangle, by straight lines parallel to the triangle sides, measuring the step $\Delta$ along a triangle side, we construct an oblique grid.

For linear and nonlinear models of the covering problem, we have calculated the number of circles of radius $r$ that cover the $1.22 \times 0.82$ rectangle so that the minimum distance between the circles' centers is not less than $r / 2$.

In Table 2, for the above-mentioned values of the covering multiplicity $(k)$ and the radius of covering circles $(r)$, we indicate consecutively: 1$)$ approximate lower bounds for the number of circles of radius $r$ in the $k$-covering of $G_{t}$ obtained with the use of LP, 2) approximate upper bounds for the number of circles of radius $r$ in the $k$-covering of $G_{t}$ obtained with the use of LP, 3) approximate upper bounds for the number of circles of radius $r$ in the $k$-covering of $G_{t}$ obtained with the use of LP and the nonlinear model of the covering problem.

Table 2. Approximate values of the number of circles in the $k$-covering of $G$

\begin{tabular}{|c|l|l|l|l|l|l|l|l|l|}
\hline \multirow{2}{*}{ Circle radius $r$} & \multicolumn{9}{|c|}{ The covering multiplicity $k$} \\
\cline { 2 - 11 } & \multicolumn{3}{|c|}{$k=1$} & \multicolumn{3}{c|}{$k=2$} & \multicolumn{3}{c|}{$k=3$} \\
\hline 0.50 & 3 & 3 & 3 & 6 & 6 & 6 & 9 & 10 & 9 \\
\hline 0.45 & 3 & 4 & 4 & 7 & 8 & 8 & 10 & 12 & 10 \\
\hline 0.40 & 4 & 4 & 4 & 8 & 8 & 8 & 12 & 13 & 12 \\
\hline
\end{tabular}

According to the data given in Table 2, the bounds obtained using the nonlinear model are in some cases better than those obtained from only the linear model. For example, the nonlinear model allows us to conclude that 10 circles of radius 0.45 are sufficient for a 3 -fold covering, while the linear model estimates this number as 12 circles.

On the left-hand side of Fig. 1, we present locations of the centers of circles of radius 0.45 that form a 3-fold covering of $G$ obtained with the use of the linear model, while on the right-hand side we do those obtained with the use of both linear and nonlinear models. In each case, the minimum distance between the circles' centers is not less than $r / 2$.

The calculations were carried out using a computer Intel Core i7-3537U, 2.5GHz, 6 GB RAM, OS Windows 10. The solution time was acceptable (it varied from several minutes to several hours); we do not give its precise values here, because in practice one usually solves the covering problems in advance (rather than online).

\section{Conclusion}

For a convex bounded plane domain $G$ with nonempty interior, we state linear and nonlinear mathematical optimization models of the multiple covering problem with circles of a given radius $r$. We establish necessary and sufficient solvability conditions for linear models introduced in a known way with the help of grids constructed on $G$. We state a nonlinear mathematical model for the $k$-covering 

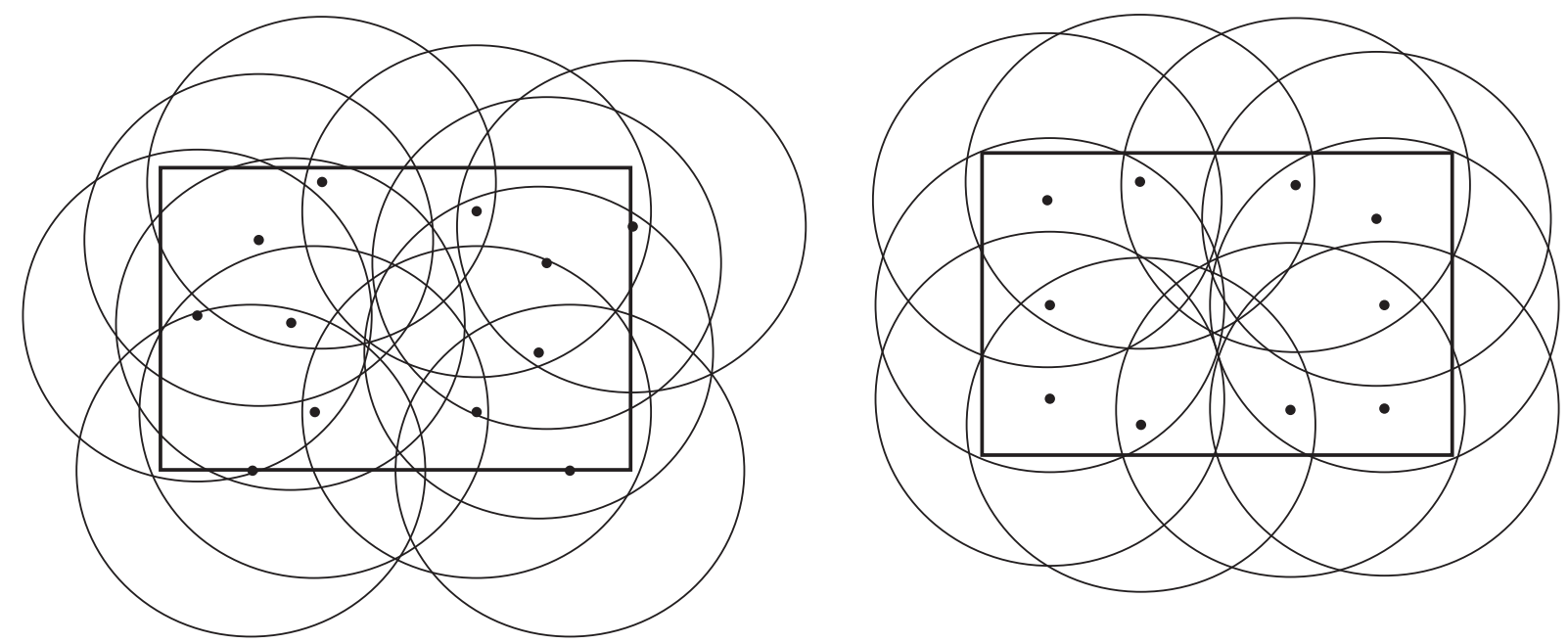

Figure 1. Locations of 12 (on the left-hand side) and 10 (on the right-hand side) circles of radius 0.45 that form a 3 -fold covering of the $1.22 \times 0.82$ rectangle

problem and propose an algorithm which (in some cases) allows one to find more exact solutions than those obtained only with the use of linear models. To implement the proposed approach, we have developed programs and performed numerical experiments. Results of numerical experiments demonstrate the effectiveness of the method.

The proposed method implying the construction of a grid on $G$ can be extended for covering problems in spaces of three and more dimensions. While solving the problem of covering with circles of a given radius $r$, we obtain an integer LP problem of the dimension $n \times n$, where $n$ is the number of nodes in the grid on $G$. In the case of covering in $s$ dimension space when $G$ is covered with balls of a given radius $r$, the dimension of the integer LP problem is $n^{s}$, provided that no constraint is imposed on the distances between the circles' centers. The solution of such problems is time-consuming and requires developing new methods for solving large-scale integer LP problems.

\section{References}

Ammari Y. M. Challenges and Opportunities of Connected k-Covered Wireless Sensor Networks. Springer-Verlag Berlin Heidelberg, 2009.

Astrakov S. N. Coverings of Sets with Restrictions on the Arrangement of Circles // Proceedings of the OPTIMA-2017 Conference. - Petrovac, Montenegro, 2017.

Culberson J. C., Reckhow R.A. Covering polygons is hard // J. Algorithms. - 1994. - Vol. 17. P. 2-44.

Erzin A., Astrakov S. Min-density stripe covering and applications in sensor networks // Lect. Notes Comput. Sci., Springer Berlin Heidelberg, Berlin, Heidelberg. - 2011. - P. 152-162.

Farahani R. Z., Asgari N., Heidari N., Hosseininia M., Goh M. Covering problems in facility location: A Review // Computers \& Industrial Engineering. - 2012. - Vol. 62. - P. 368-407.

Huang C.F., Tseng Y.C. A Survey of Solutions to the Coverage Problems in Wireless Sensor Networks // J. Internet Technol. - 2005. - Vol. 6, No. 1. - P. 1-8.

Galiev Sh. I. The Direction of Decrease for Minmaxmin Problems // Computational Mathematics and Mathematical Physics. - 1994. - Vol. 34, No. 3. - P. 271-286.

Galiev. Sh. I. Napravleniya ubyvaniya dlya minimaksiminnykh zadach [The Direction of Decrease for Minmaxmin Problems] // Zhurnal vychisl. matem. i matem. fiz. - 1994. - Vol. 34, No. 3. - P. 323-343 (in Russian). 
Galiev Sh.I., Karpova M.A. Optimization of Multiple Covering of a Bounded Set with Circles // Comput. Math. Math. Phys. - 2010. - Vol. 50. - P. 721-732.

Galiev. Sh.I., Karpova M.A. Optimizatsiya mnogokratnogo pokrytiya ogranichennogo mnozhestva krugami [Optimization of multiple coverage of a bounded set of circles] // Zhurnal vychisl. matem. i matem. fiz. - 2010. Vol. 50, No. 7. - P. 757-769 (in Russian).

Galiev Sh. I., Lisafina M. S. Linear models for the approximate solution of the problem of packing equal circles into a given domain // European Journal of Operational Research. - 2013. - Vol. 230. P. 505-514.

Galiev Sh.I., Khorkov A. V. On the Number and Arrangement of Sensors for Multiple Covering of Bounded Plane Domains // Journal of Applied and Industrial Mathematics. - 2019. - Vol. 13, No. 1. - P. 43-53.

Galiev. Sh. I., Khorkov A.V. O chisle i raspolozhenii sensorov dlya mnogokratnogo pokrytiya ogranichennoi chasti ploskosti [On the Number and Arrangement of Sensors for Multiple Covering of Bounded Plane Domains] // Discrete analysis and operations research. - 2019. - Vol. 26, No. 1. - P. 33-53 (in Russian).

Garey M. R., Johnson D. S. Computers and Intractability: A Guide to the Theory of NP-completeness. San Francisco: W. H. Freeman, 1979. - 338 p.

Khachai M.Yu., Poberiy M.I. The computational complexity and approximability of a series of geometric covering problems // Proceedings of the Steklov Institute of Mathematics. - 2013. Vol. 283, No. 1. - P. 64-77.

Kim J. E., Han J., Lee C. G. Optimal 3-coverage with minimum separation requirements for ubiquitous computing environments // Mobile Netw. Appl. - 2009. - P. 556-570.

Megiddo N. On the complexity of some common geometric location problems // SIAM J. Comput. 1984. - Vol. 13. - P. 182-196.

Nurmella K. J. Conjecturally optimal coverings of an equilateral triangle with up to 36 equal circles // Exp. Math. - 2000. - Vol. 9, No. 2. - P. 241-250.

Peixoto J.P. J., Costa D. G. Wireless visual sensor networks for smart city applications: A relevancebased approach for multiple sinks mobility // Future Generation Computer Systems. - 2017. Vol. 76. - P. 51-62.

Suzuki A., Drezner Z. The minimum number equitable radius location problems with continuous domain // Eur. J. Oper. Res. - 2009. - P. 17-30.

Tabirca T., Yang L.T., Tabirca S. Smallest number of sensors for k-covering // Int. J. Comput. Commun. - 2013. - Vol. 8. - P. 312-319.

Wang B. Coverage problem in sensor networks: a survey // ACM computing surveys. - 2011. Vol. 43, No. 4, Article 32.

Yeasmin N. k-Coverage Problems and Solutions in Wireless Sensor Networks: A Survey // Internat. J. Comput. Appl. - 2014. - Vol. 100, No. 17. - P. 1-6.

Zhang C., Zhang Y., Fang Y. Localized algorithms for coverage boundary detection in wireless sensor networks // Wireless Networks. - 2009. - Vol. 15, No. 1. - P. 3-20. 